\title{
The Mechanistic Role of Proton-Hydride Pair in Heteroarene Catalytic Hydrogenation
}

\author{
Haiting Cai, ${ }^{\S, 1}$ Roberto Schimmenti, ${ }^{\S, 2}$ Haoyu Nie, ${ }^{1}$ Manos Mavrikakis, ${ }^{*, 2}$ Ya-Huei (Cathy) Chin $*, 1$ \\ ${ }^{1}$ Department of Chemical Engineering and Applied Chemistry, University of Toronto, Toronto, Ontario M5S \\ 3E5, Canada \\ ${ }^{2}$ Department of Chemical and Biological Engineering, University of Wisconsin-Madison, Madison, Wisconsin \\ 53706, USA
}

\section{Corresponding Authors: \\ *cathy.chin@utoronto.ca \\ *emavrikakis@wisc.edu \\ Author Contributions}

${ }^{\S}$ These authors contributed equally. 


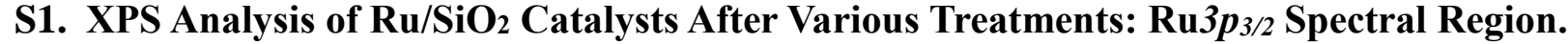

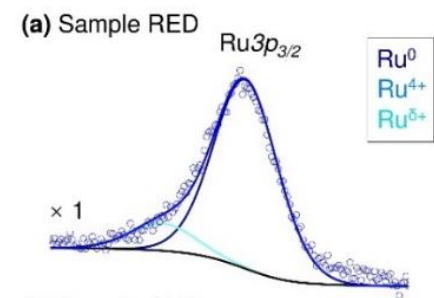

(b) Sample SHP

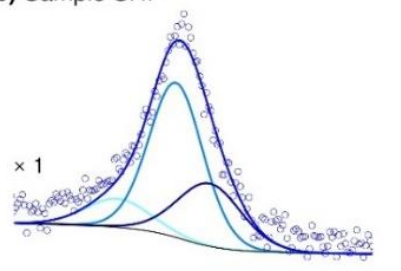

(c) Sample RXN

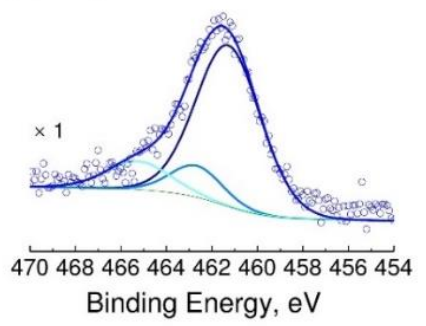

Figure S1. High-resolution XPS spectra in the region of $\mathrm{Ru} 3 p_{3 / 2}$ of $\mathrm{Ru} / \mathrm{SiO}_{2}$ samples after the following treatments: (a) reduction in $101 \mathrm{kPa} \mathrm{H}_{2}$ at $623 \mathrm{~K}$ (Sample RED), (b) sulfidation in $10.1 \mathrm{kPa} \mathrm{H}_{2} \mathrm{~S}$ and $90.9 \mathrm{kPa} \mathrm{He}$ at $673 \mathrm{~K}$, after reduction in $101 \mathrm{kPa} \mathrm{H}_{2}$ at $623 \mathrm{~K}$ (Sample SHP), and (c) hydrogenation in $1.8 \mathrm{kPa} \mathrm{H}_{2} \mathrm{~S}, 1200 \mathrm{kPa} \mathrm{H}_{2}$, and $6.0 \mathrm{kPa}$ pyridine at $573 \mathrm{~K}$, after reduction in $101 \mathrm{kPa} \mathrm{H}_{2}$ at $623 \mathrm{~K}$ and then sulfidation in $0.15 \mathrm{kPa} \mathrm{H} \mathrm{H}_{2} \mathrm{~S}, 99.50 \mathrm{kPa} \mathrm{H}_{2}$, and $1.35 \mathrm{kPa} \mathrm{He}$ at $673 \mathrm{~K}$ (Sample RXN).

Figure $\mathrm{S} 1$ shows the Ru3p3/2 XPS spectra for the $\mathrm{Ru} / \mathrm{SiO}_{2}$ samples with bands at 461.2, 462.9, and 465.1 eV, which are assigned to $\mathrm{Ru}^{0}, \mathrm{Ru}^{4+}$, and highly oxidized $\mathrm{Ru}^{\delta+}$ state, respectively. All samples contain similar amounts of irreducible $\mathrm{Ru}^{\delta+}$ cations bound to oxygen species (i.e., 12.6\%, 12.8\%, and 12.9\% for Sample RED, RXN, and SHP, respectively). The $\mathrm{Ru}^{4+}$-to-total $\mathrm{Ru}$ ratio in Sample SHP is 0.59, higher than that in Sample RXN, which is 0.12. The calculation based on the amount of $\mathrm{Ru}^{4+}$ in each sample shows that the former forms four monolayers of $\mathrm{RuS}_{2}$ and the latter forms one monolayer of $\mathrm{RuS}_{2}{ }^{1}$

The number of near-surface $\mathrm{RuS}_{2}$ layers was determined with the assumption of truncated hexagonal bipyramid shape of sulfided Ru clusters. $N_{R u_{\text {total red }}}$ is the total number of $\mathrm{Ru}$ atoms in a Ru cluster and varies with the number of atom layers, $m_{\text {total }}$. When $m_{\text {total }}$ is an even number:

$N_{R u_{\text {total red }}}=\frac{14 m_{\text {total }^{3}}-21 m_{\text {total }^{2}}+14 m_{\text {total }}-4}{4}$

Alternatively, when $m_{\text {total }}$ is an odd number: 
$N_{R u_{\text {total red }}}=\frac{14 m_{\text {total }}{ }^{3}-21 m_{\text {total }^{2}}+14 m_{\text {total }}-3}{4}$

The number of $\mathrm{Ru}^{0}, N_{R u^{0}}$, is calculated via:

$N_{R u^{0}}=\frac{N_{R u_{\text {total red }} I_{R u^{0}}}}{I_{R u_{\text {total red }}}}$

in which $I_{R u^{0}}$ and $I_{R u_{\text {total red }}}$ represent intensities of bands for $\mathrm{Ru}^{0}$ and reducible $\mathrm{Ru}$ species $\left(\mathrm{Ru}^{0}\right.$ and $\left.\mathrm{Ru}^{4+}\right)$ in the XPS spectra, respectively. The number of $\mathrm{Ru}^{0}$ layers in the bulk of each sulfided $\mathrm{Ru}$ cluster, $m_{R u^{0}}$, is given by the following two cases of:

Case 1. When $m_{R u^{0}}$ is an even number:

$N_{R u^{0}}=\frac{14 m_{R u^{0}}{ }^{3}-21 m_{R u^{0}}{ }^{2}+14 m_{R u^{0}}-4}{4}$

Case 2. When $m_{R u^{0}}$ is an odd number:

$N_{R u^{0}}=\frac{14 m_{R u^{0}}{ }^{3}-21 m_{R u^{0}}{ }^{2}+14 m_{R u^{0}}-3}{4}$

The number of near-surface $\mathrm{RuS}_{2}$ layers, $m_{R u S_{2}}$, is determined by:

$m_{R u S_{2}}=m_{\text {total }}-m_{R u^{0}}$

The calculated values of the parameters in Equations S1-S5 for the catalyst samples after the various treatments are summarized in Table S1.

Table S1. Values of Parameters in the Calculation of Near-surface $\mathrm{RuS}_{2}$ Layers in Each Sulfided Ru Cluster

\begin{tabular}{cccc}
\hline Parameter & Sample SLP & Sample SHP & Sample RXN \\
\hline$I_{R u^{0}} / I_{R u_{\text {total }}}$ & 0.91 & 0.43 & 0.83 \\
$N_{R u_{\text {total }}}$ & 7970 & 7970 & 7970 \\
$N_{R u^{0}}$ & 7230 & 3395 & 6634 \\
$m_{\text {total }}$ & 14 & 14 & 14 \\
$m_{R u^{0}}$ & 13 & 10 & 13 \\
$m_{R u S_{2}}$ & 1 & 4 & 1 \\
\hline
\end{tabular}

\section{S2. Sensitivity Analyses on the Effective Rate Constants and Apparent Orders.}

The apparent orders of pyridine and pyrrole hydrogenation in Equation 11 and 12 are obtained from the nonlinear regressions of the experimental turnover rates $\left(r_{H Y D, P Y R}\right.$ for pyridine hydrogenation, and $r_{H Y D, P Y L}$ for pyrrole hydrogenation) with Equation S6 and S7, which are the turnover rate expressions for pyridine and pyrrole 
hydrogenation, respectively. The exponents $a_{j}, b_{j}$, and $c_{j}$ represent the apparent orders of $\mathrm{H}_{2}, \mathrm{H}_{2} \mathrm{~S}$, and pyridine/pyrrole with respect to $j$ species $(j=P Y R$ or $P Y L)$ in the hydrogenation of $j ; k_{P Y R, e f f}$ and $k_{P Y L, e f f}$ are the effective rate constants for the hydrogenation of pyridine or pyrrole, respectively. The solid lines in Figures S2 and S3 are the optimized fittings predicted from Equation S6 and S7, respectively, with the optimized values of $k_{P Y R, e f f}, k_{P Y L, e f f}, a_{j}, b_{j}$, and $c_{j}$ shown in Table S2. Figure S4 presents the sensitivity analyses performed by introducing changes $( \pm 25 \%)$ to these optimal parameters.

$$
\begin{aligned}
& r_{H Y D, P Y R}=k_{P Y R, e f f}\left[H_{2}\right]^{a_{P Y R}}\left[H_{2} S\right]^{b_{P Y R}[P Y R]^{c_{P Y R}}} \\
& r_{H Y D, P Y L}=k_{P Y L, e f f}\left[H_{2}\right]^{a_{P Y L}\left[H_{2} S\right]^{b_{P Y L}}[P Y L]^{c_{P Y L}}}
\end{aligned}
$$
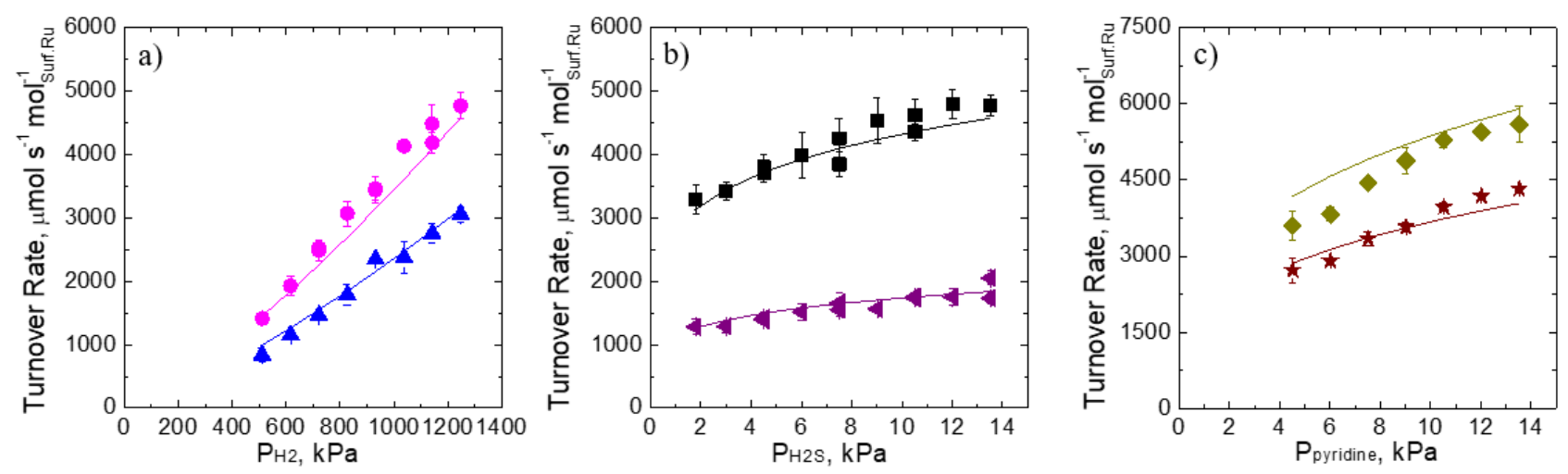

Figure S2. Effect of (a) $\mathrm{H}_{2}$, (b) $\mathrm{H}_{2} \mathrm{~S}$, and (c) pyridine partial pressures for pyridine HYD on RuS $\mathrm{S}_{\mathrm{x}}$ surfaces at $573 \mathrm{~K}$ and 1.5 MPa with $1.8 \mathrm{kPa} \mathrm{H} 2 \mathrm{~S}(\star, \Delta), 13.5 \mathrm{kPa} \mathrm{H}_{2} \mathrm{~S}(\bullet, \bullet), 616 \mathrm{kPa} \mathrm{H}_{2}(\triangleleft), 1250 \mathrm{kPa} \mathrm{H}_{2}(\star, \bullet, \mathbf{\square})$, and $6.0 \mathrm{kPa}$ pyridine $(\mathbf{\bullet}, 4$, $\Delta, \bullet), 0.045 \mathrm{~m}^{3} \mathrm{~h}^{-1} \mathrm{~g}_{\text {cat }}{ }^{-1}$. Solid lines are the power law fittings predicted from Equation $\mathrm{S} 6$.
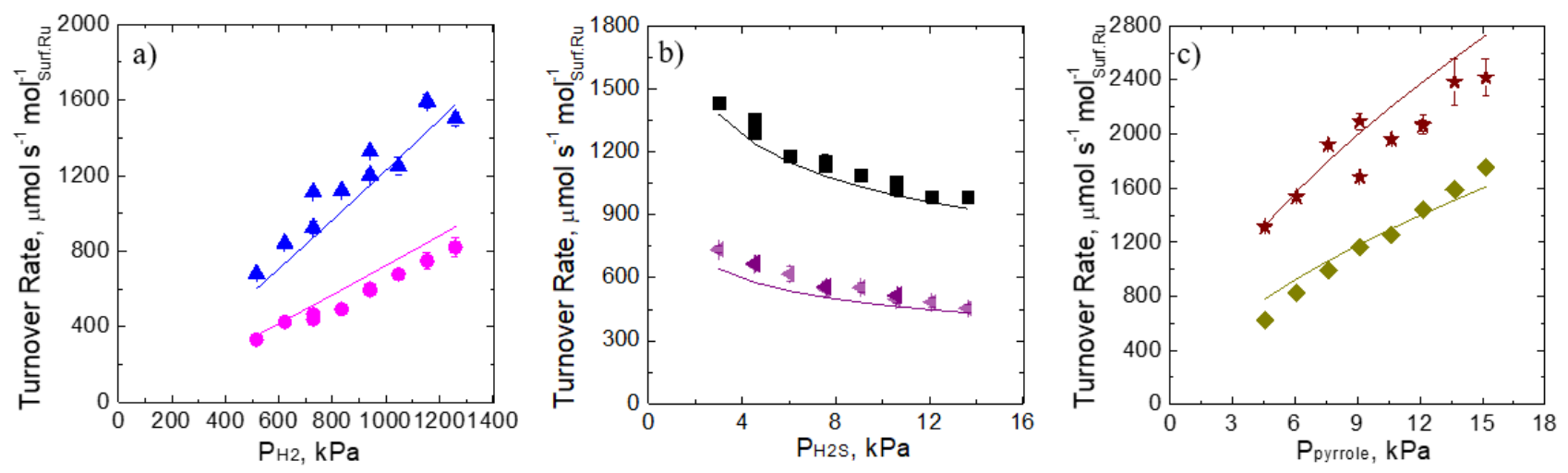

Figure S3. Effect of (a) $\mathrm{H}_{2}$, (b) $\mathrm{H}_{2} \mathrm{~S}$, and (c) pyrrole partial pressures for pyrrole HYD on $\mathrm{RuS}_{\mathrm{x}}$ surfaces at $573 \mathrm{~K}$ and 1.5

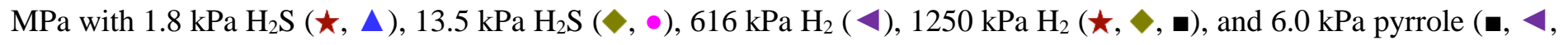
$\Delta, \bullet), 0.045 \mathrm{~m}^{3} \mathrm{~h}^{-1} \mathrm{~g}_{\text {cat }}{ }^{-1}$. Solid lines are the power law fittings predicted from Equation S7. 
Table S2. Effective Rate Constants and Apparent Orders of Pyridine and Pyrrole Hydrogenation on RuS $\mathrm{S}_{\mathrm{x}}$ Covered Ru Clusters at 573 K, Predicted from Equation S6 and S7

\begin{tabular}{|c|c|c|c|c|}
\hline & $k_{P Y R, \text { eff }}$ or $k_{P Y L, e f f}$, & $a_{j}$ & $c_{j}$ \\
\hline $\begin{array}{c}\text { Pyridine } \\
\text { Hydrogenation }\end{array}$ & $0.15 \pm 0.04$ & $1.30 \pm 0.04$ & $0.19 \pm 0.02$ & $0.31 \pm 0.06$ \\
\hline $\begin{array}{c}\text { Pyrrole } \\
\text { Hydrogenation }\end{array}$ & $0.28 \pm 0.09$ & $1.08 \pm 0.05$ & $-0.26 \pm 0.01$ & $0.60 \pm 0.05$ \\
\hline
\end{tabular}
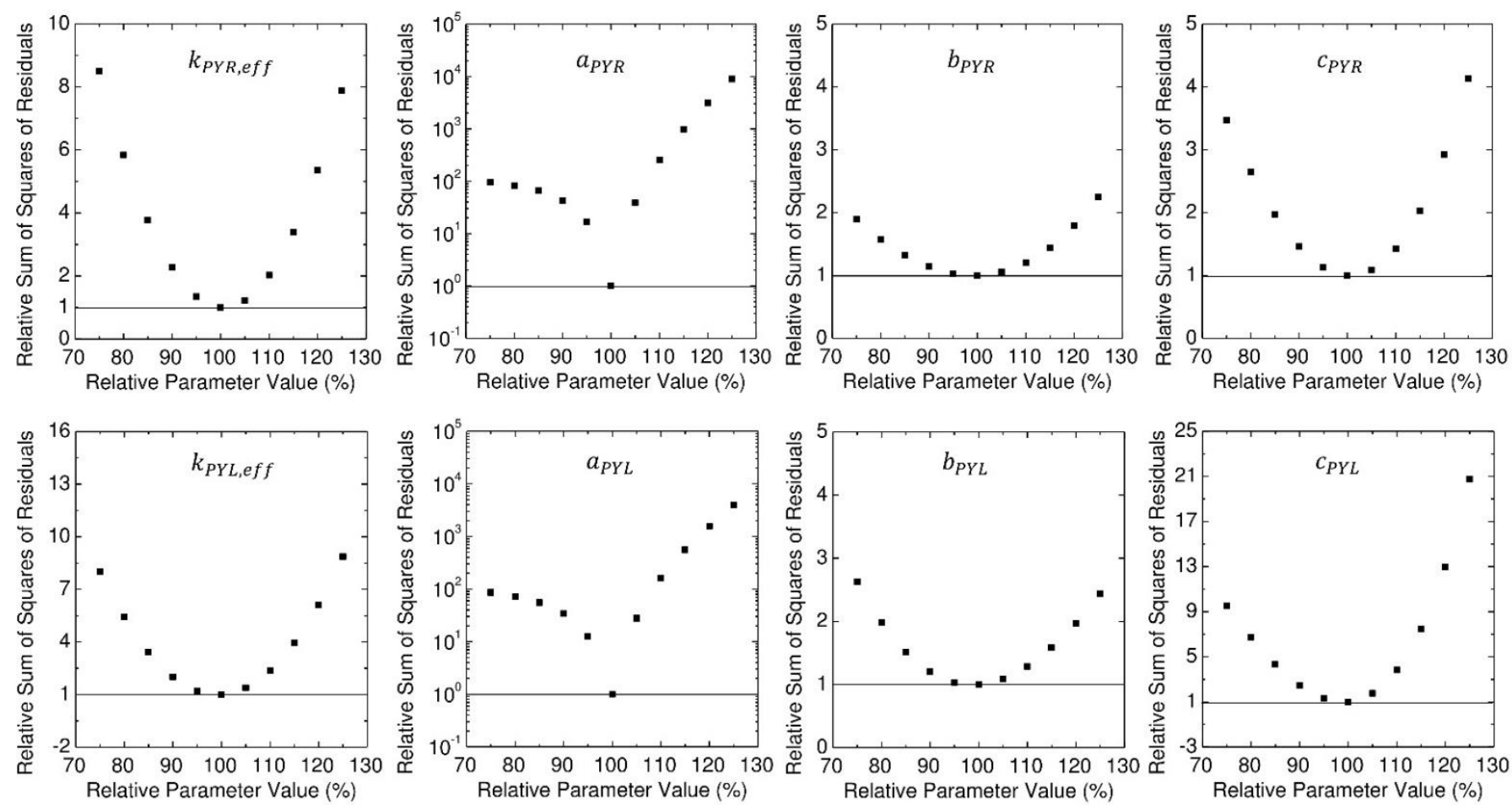

Figure S4. Sensitivity of sum of squares of residuals to variations in optimal parameters of the turnover rate expressions of pyridine and pyrrole hydrogenation (Equation $\mathrm{S} 6$ and S7) on $5.9 \mathrm{~nm} \mathrm{RuS} / \mathrm{SiO}_{2}$ catalyst at $573 \mathrm{~K}$.

\section{S3. Derivation of the Rate Expressions for Pyridine and Pyrrole Hydrogenation on Sulfided Ru Clusters.}

The overall turnover rate of pyridine $\left(r_{H Y D, P Y R}\right.$, per exposed $\mathrm{Ru}$ atom) is derived from the catalytic sequence proposed in Scheme 4 of the main manuscript, by considering the $a^{\text {th }}(1 \leq a \leq 6)$ hydrogen addition via PYR-A and the $c^{\text {th }}(1 \leq c \leq 6)$ hydrogen addition via PYR-C, both in Scheme 4 of the main manuscript, as the kinetically relevant steps and by applying pseudo steady-state assumptions on all reactive intermediates. It equals the sum of the individual turnover rate of each pathway:

$r_{H Y D, P Y R}=r_{H Y D, P Y R, A}+r_{H Y D, P Y R, C}$ 
$r_{H Y D, P Y R}=\frac{k_{H Y D, P Y R, A}\left[R u^{4+}-\left(H^{\delta-}\right)-S^{2-}-\left(H^{\delta+}\right)\right]\left[R u^{4+}-\left(S H_{2}-P Y R\right)-S^{2-}\right]_{a=1}\left[R u^{4+}{ }_{-}\left(H^{\delta-}\right)-S^{2-}-\left(H_{n-1} P Y R\right)\right]_{n=a, a=2,3}\left[R u^{4+}{ }_{-} S^{2-}-\left(H_{n-1} P Y R\right)\right]_{n=a, a \geq 4}}{\left[T_{P Y R}\right]^{2}}+$

$\frac{k_{H Y D, P Y R, C}\left[R u^{4+}-\left(S H_{2}-P Y R\right)-S^{2-}\right]_{c=1}\left[R u^{4+}-\left(H^{\delta-}\right)-S^{2-}-\left(H^{\delta+}\right)\right]_{c=2,4,6}\left[R u^{4+}-\left(S H_{2}\right)-S^{2-}\right]_{c=3,5}\left[R u^{4+}-(S H-H P Y R)-S^{2-}\right]_{c=2}\left[R u^{4+}-S^{2-}-\left(H_{n}{ }_{-1} P Y R\right)\right]_{n}{ }_{n}=c, c \geq 3}{\left[T_{P Y R}\right]_{c=1} \text { or }\left[T_{P Y R}\right]_{c \geq 2}^{2}}$

(a) Pyridine Hydrogenation

PYR-A: Pyridine Hydrogenation via Path 2A1

Step PYR-A(i): Pyridine Adsorption

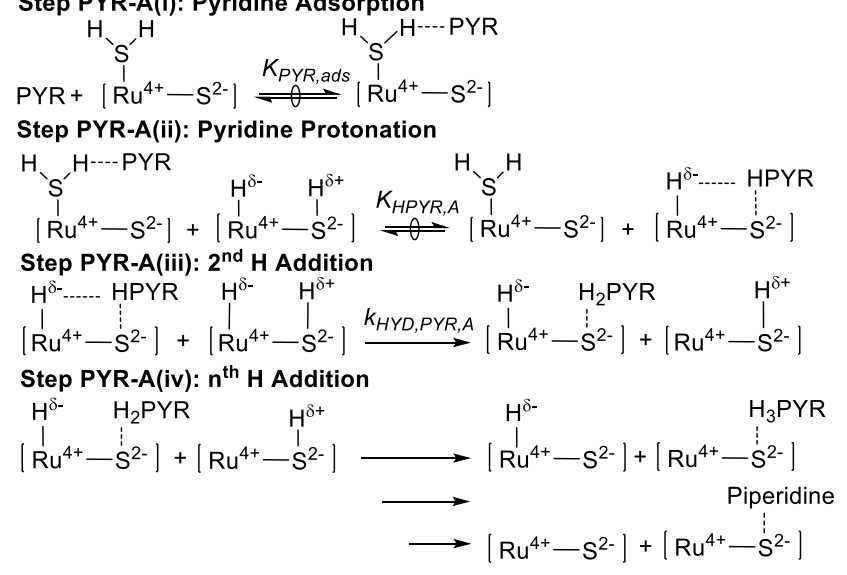

PYR-C: Pyridine Hydrogenation via Path 2C1

Step PYR-C(i) Pyridine Protonation

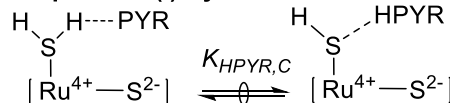

Step PYR-C(ii): $2^{\text {nd }} H$ Addition

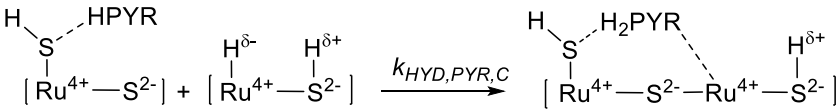

Step PYR-C(iii): S-N Cleavage

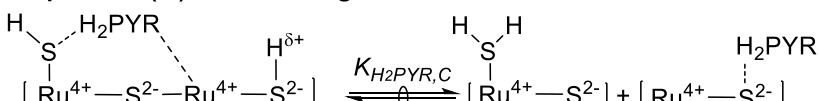

Step PYR-C(iv): $\mathbf{n}^{\text {th }} \mathrm{H}$ Addition

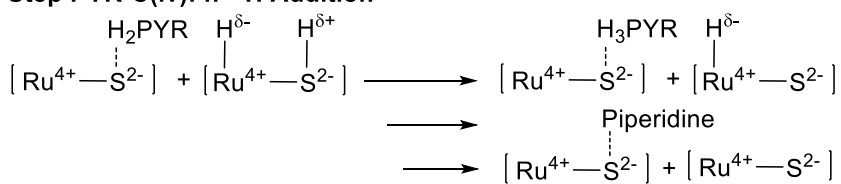

Step PYR-C(v): Piperidine Desorption

$\left[\mathrm{Ru}^{4+}-\mathrm{S}^{2-} \mid \stackrel{\text { Piperidine }}{\longrightarrow}\right.$ Piperidine $+\left[\mathrm{Ru}^{4+}-\mathrm{S}^{2-} \mid\right.$

\section{(b) Pyrrole Hydrogenation}

PYL-B: Pyrrole Hydrogenation via Path $3 \mathrm{~B}$

Step PYL-B(iA): Pyrrole Adsorption on $\mathrm{Ru}^{4+}-\left(\mathrm{SH}_{2}\right)-\mathrm{S}^{2-}$

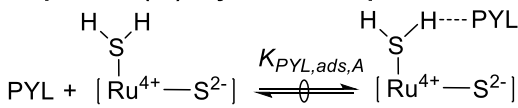

Step PYL-B(iiA): Pyrrole Protonation

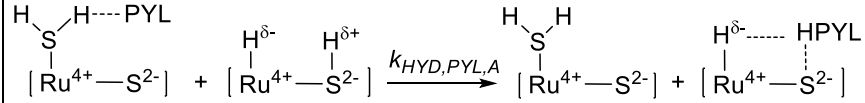

Step PYL-B(iB): Pyrrole Adsorption on $\mathrm{Ru}^{4+}-\mathrm{S}^{2-}$

$\mathrm{PYL}+\left[\mathrm{Ru}^{4+}-\mathrm{S}^{2-}\right] \stackrel{\mathrm{C} Y \mathrm{KYL,ads,B}}{=}\left[\mathrm{Ru}^{4+}-\mathrm{S}^{2-}\right]$

Step PYL-B(iiB): Pyrrole Protonation

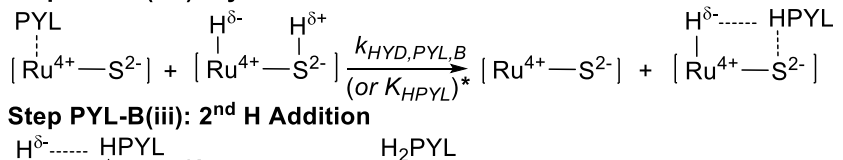

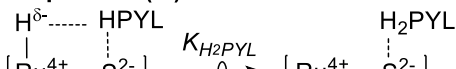

$\left[\mathrm{Ru}^{4+}-\mathrm{S}^{2-}\right] \stackrel{\mathrm{H}^{\mathrm{H} 2 P Y L}}{=}\left[\mathrm{Ru}^{4+}-\mathrm{S}^{2-}\right]$

Step PYL-B(iv): $\mathbf{n}^{\text {th }} \mathbf{H}$ Addition

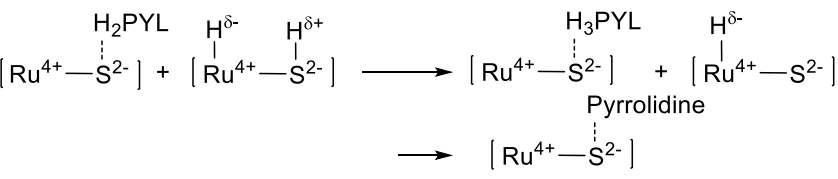

Step PYL-B(v): Pyrrolidine Desorption

Pyrrolidine

$\left[\mathrm{Ru}^{4+}-\mathrm{S}^{2-}\right] \stackrel{K_{\text {pyrrolidine }}}{=}$ Pyrrolidine+ $\left[\mathrm{Ru}^{4+}-\mathrm{S}^{2-}\right]$

Scheme S1. Proposed elementary steps for (a) pyridine and (b) pyrrole hydrogenation on $R u S_{x}$ surfaces. " $\longrightarrow$ " represents an irreversible reaction, " $\rightleftharpoons$ " denotes a quasi-equilibrated reaction, and " $\rightarrow$ " gives the electron transfer direction. $K_{j}$ is the equilibrium constant for each elementary step, where subscript $j$ represents the $\mathrm{H}_{2}, \mathrm{H}_{2} \mathrm{~S}$, or $\mathrm{N}$-containing reactant of each elementary step; $k_{H Y D, i, L}$ denotes the rate constant for the kinetically relevant step of pyridine or pyrrole hydrogenation, where subscript $H Y D, i, L$ represents pyridine or pyrrole ( $i=$ pyridine or pyrrole) hydrogenation via Path $\mathrm{L}(\mathrm{L}=\mathrm{A}, \mathrm{B}$, or $\mathrm{C})$. The species in a same bracket represents a reactive couple. "If this step is quasi-equilibrated, the equilibrium constant is $K_{H P Y L}$ 
$r_{H Y D, P Y R, A}\left(k_{H Y D, P Y R, A}\right)$ and $r_{H Y D, P Y R, C}\left(k_{H Y D, P Y R, C}\right)$ are the turnover rates (rate constants) of pyridine hydrogenation via Scheme 4 PYR-A and PYR-C, respectively. $\left[T_{P Y R}\right]$ denotes the total surface sites during pyridine hydrogenation reaction. We assume that the coverage of the $\mathrm{N}$-containing intermediates generated after the rate determining step remains insignificant. Therefore, the reactive couple $R u^{4+}-S^{2-}$ remains either unoccupied or dominated by $R u^{4+}-\left(H^{\delta-}\right)-S^{2-}-\left(H^{\delta+}\right), R u^{4+}-\left(S H_{2}\right)-S^{2-}, R u^{4+}-\left(S H_{2}-P Y R\right)-S^{2-}$, $\left[R u^{4+}-\left(H^{\delta-}\right)-S^{2-}-\left(H_{n-1} P Y R\right)\right]_{n=a, a=2,3}$, and $R u^{4+}-(S H-H P Y R)-S^{2-},\left[R u^{4+}-S^{2-}-\left(H_{n-1} P Y R\right)\right]_{n=a, a \geq 4}$, and $\left[R u^{4+}-S^{2-}-\left(H_{n^{\prime}-1} P Y R\right)\right]_{n^{\prime}=c, c \geq 3}$ species. As a result, the total sites can be denoted as:

$\left[T_{P Y R}\right]=\left[R u^{4+}-S^{2-}\right]+\left[R u^{4+}-\left(H^{\delta-}\right)-S^{2-}-\left(H^{\delta+}\right)\right]+\left[R u^{4+}-\left(S_{2}\right)-S^{2-}\right]+\left[R u^{4+}-\left(S_{2}-P Y R\right)-S^{2-}\right]+$ $\left[R u^{4+}-\left(H^{\delta-}\right)-S^{2-}-\left(H_{n-1} P Y R\right)\right]_{n=a, a=2,3}+\left[R u^{4+}-(S H-H P Y R)-S^{2-}\right]+\cdots+\left[R u^{4+}-S^{2-}-\left(H_{n-1} P Y R\right)\right]_{n=a, a \geq 4}+$ $\left[R u^{4+}-S^{2-}-\left(H_{n^{\prime}-1} P Y R\right)\right]_{n^{\prime}=c, c \geq 3}$

The relative abundances of surface species and vacancies are dictated by equilibrium constants of the elementary steps (Schemes 1a and 4), and the partial pressures of $\mathrm{H}_{2}, \mathrm{H}_{2} \mathrm{~S}$, and pyridine.

$$
\begin{aligned}
& {\left[R u^{4+}-\left(H^{\delta-}\right)-S^{2-}-\left(H^{\delta+}\right)\right]=K_{H 2}\left[H_{2}\right]\left[R u^{4+}-S^{2-}\right]} \\
& {\left[R u^{4+}-\left(S H_{2}\right)-S^{2-}\right]=K_{H 2 S}\left[H_{2} S\right]\left[R u^{4+}-S^{2-}\right]} \\
& {\left[R u^{4+}-\left(S H_{2}-P Y R\right)-S^{2-}\right]=K_{P Y R, a d s}[P Y R] K_{H 2 S}\left[H_{2} S\right]\left[R u^{4+}-S^{2-}\right]} \\
& {\left[R u^{4+}-\left(H^{\delta-}\right)-S^{2-}-(H P Y R)\right]=K_{H P Y R, A} K_{P Y R, a d s}[P Y R] K_{H 2}\left[H_{2}\right]\left[R u^{4+}-S^{2-}\right]} \\
& {\left[R u^{4+}-(S H-H P Y R)-S^{2-}\right]=K_{H P Y R, C} K_{P Y R, a d s}[P Y R] K_{H 2 S}\left[H_{2} S\right]\left[R u^{4+}-_{-}^{2-}\right]} \\
& {\left[R u^{4+}-\left(H^{\delta-}\right)-S^{2-}-\left(H_{2} P Y R\right)\right]=K_{H 2 P Y R, A} K_{H P Y R, A} K_{P Y R, a d s}[P Y R]\left(K_{H 2}\left[H_{2}\right]\right)^{1.5}\left[R u^{4+}-S^{2-}\right]} \\
& {\left[R u^{4+}-S^{2-}-\left(H_{n-1} P Y R\right)\right]=K_{H_{n-1} P Y R, A} K_{H_{n-2} P Y R, A} \cdots K_{H P Y R, A} K_{P Y R, a d s}[P Y R]\left(K_{H 2}\left[H_{2}\right]\right)^{0.5 n-0.5}\left[R u^{4+}-S^{2-}\right]} \\
& {\left[R u^{4+}-S^{2-}-\left(H_{n \prime-1} P Y R\right)\right]=K_{H_{n \prime-1} P Y R, C} K_{H_{n \prime-2} P Y R, C} \cdots K_{H P Y R, C} K_{P Y R, a d s}\left(K_{H 2}\left[H_{2}\right]\right)^{0.5 n \prime-0.5}\left[R u^{4+}-S^{2-}\right]}
\end{aligned}
$$

The concentrations of surface intermediates are expressed in terms of reactive couple $\left(\left[R u^{4+}-S^{2-}\right]\right)$ using quasiequilibrium approximations. Substituting Equations S11-S18 into Equation S10 gives:

$$
\begin{aligned}
\frac{\left[T_{P Y R}\right]}{\left[R u^{4+}-S^{2-}\right]} & =1+K_{H 2}\left[H_{2}\right]+K_{H 2 S}\left[H_{2} S\right]+K_{P Y R, a d s}[P Y R] K_{H 2 S}\left[H_{2} S\right]+K_{H P Y R, A} K_{P Y R, a d s}[P Y R] K_{H 2}\left[H_{2}\right] \\
& +K_{H P Y R, C} K_{P Y R, a d s}[P Y R] K_{H 2 S}\left[H_{2} S\right]+K_{H 2 P Y R, A} K_{H P Y R, A} K_{P Y R, a d s}[P Y R]\left(K_{H 2}\left[H_{2}\right]\right)^{1.5} \\
& +\cdots+\left\{K_{H_{n-1} P Y R, A} K_{H_{n-2} P Y R, A} \cdots K_{H P Y R, A} K_{P Y R, a d s}[P Y R]\left(K_{H 2}\left[H_{2}\right]\right)^{0.5 n-0.5}\right\}_{n=a, a \geq 4}
\end{aligned}
$$




$$
+\left\{K_{H_{n \prime-1} P Y R, C} K_{H_{n^{\prime}-2} P Y R, C} \cdots K_{H P Y R, C} K_{P Y R, a d s}\left(K_{H 2}\left[H_{2}\right]\right)^{0.5 n^{\prime}-0.5}\right\}_{n^{\prime}=c, c \geq 3}
$$

If $\theta=1+K_{H_{2}}\left[H_{2}\right]+K_{H_{2} S}\left[H_{2} S\right]$, and

$$
\begin{aligned}
\varphi_{P Y R}= & K_{P Y R, a d s}[P Y R] K_{H 2 S}\left[H_{2} S\right]+K_{H P Y R, A} K_{P Y R, a d s}[P Y R] K_{H 2}\left[H_{2}\right]+K_{H P Y R, C} K_{P Y R, a d s}[P Y R] K_{H 2 S}\left[H_{2} S\right] \\
& +K_{H 2 P Y R, A} K_{H P Y R, A} K_{P Y R, a d s}[P Y R]\left(K_{H 2}\left[H_{2}\right]\right)^{1.5} \\
& +\cdots+\left\{K_{H_{n-1} P Y R, A} K_{H_{n-2} P Y R, A} \cdots K_{H P Y R, A} K_{P Y R, a d s}[P Y R]\left(K_{H 2}\left[H_{2}\right]\right)^{0.5 n-0.5}\right\}_{n=a, a \geq 4} \\
& +\left\{K_{H_{n^{\prime}-1} P Y R, C} K_{H_{n^{\prime}-2} P Y R, C} \cdots K_{H P Y R, C} K_{P Y R, a d s}\left(K_{H 2}\left[H_{2}\right]\right)^{0.5 n^{\prime}-0.5}\right\}_{n^{\prime}=c, c \geq 3}
\end{aligned}
$$

Substituting Equations S11-S21 into Equation S9 leads to the final expression for the overall pyridine turnover rate $\left(r_{H Y D, P Y R}\right)$ :

$$
\begin{aligned}
& r_{H Y D, P Y R}=\frac{k_{H Y D, P Y R, A}\left\{\left(K_{H_{n-1} P Y R, A} K_{H_{n-2} P Y R, A} \cdots K_{H P Y R, A}\right)_{n=a, a \geq 2} K_{P Y R, a d s}\right\}[P Y R]\left(K_{H_{2} S}\left[H_{2} S\right]\right)_{a \leq 2}^{2-a}\left\{\left(K_{H_{2}}\left[H_{2}\right]\right)_{a=1}\left(K_{H_{2}}\left[H_{2}\right]\right)_{a=2,3}^{0.5 a+1}\left(K_{H_{2}}\left[H_{2}\right]\right)_{a \geq 4}^{0.5 a+0.5}\right\}}{\left(\theta+\varphi_{P Y R}\right)^{2}} \\
& +\frac{k_{H Y D, P Y R, C}\left\{\left(K_{H_{n-1} P Y R, C} K_{H_{n-2} P Y R, C} \cdots K_{H P Y R, C}\right)_{n=c, c \geq 2} K_{P Y R, a d s}\right\}[P Y R]\left(K_{H_{2} S}\left[H_{2} S\right]\right)_{c=1,2,3,5}\left\{\left(K_{H_{2}}\left[H_{2}\right]\right)_{c=1,2}^{c-1}\left(K_{H_{2}}\left[H_{2}\right]\right)_{c=3,5}^{0.5 c-0.5}\left(K_{H_{2}}\left[H_{2}\right]\right)_{c=4,6}^{0.5 c+0.5}\right\}}{\left\{\theta+\varphi_{P Y R}\right\}_{c=1} \text { or }\left\{\left(\theta+\varphi_{P Y R}\right)^{2}\right\}_{c \geq 2}}
\end{aligned}
$$

when $1 \leq a \leq 2$ and $1 \leq c \leq 2$, the generalized turnover rate expression can be simplified into:

$$
\begin{gathered}
r_{H Y D, P Y R}=\frac{k_{H Y D, P Y R, A}\left\{\left(K_{H_{n-1} P Y R, A} K_{H_{n-2} P Y R, A} \cdots K_{H P Y R, A}\right)_{n=a, a \geq 2} K_{P Y R, a d s}\right\}[P Y R]\left(K_{H_{2} S}\left[H_{2} S\right]\right)_{a \leq 2}^{2-a}\left(K_{H_{2}}\left[H_{2}\right]\right)^{a}}{\left(\theta+\varphi_{P Y R}\right)^{2}} \\
+\frac{k_{H Y D, P Y R, C}\left\{\left(K_{H_{n-1} P Y R, C} K_{H_{n-2} P Y R, C} \cdots K_{H P Y R, C}\right)_{n=c, c \geq 2} K_{P Y R, a d s}\right\}[P Y R] K_{H_{2} S}\left[H_{2} S\right]\left(K_{H_{2}}\left[H_{2}\right]\right)^{c-1}}{\left\{\theta+\varphi_{P Y R}\right\}_{c=1} \operatorname{or}\left\{\left(\theta+\varphi_{P Y R}\right)^{2}\right\}_{c \geq 2}}
\end{gathered}
$$

which is also Equation 17 in the main manuscript.

Likewise, the overall turnover rate of pyrrole $\left(r_{H Y D, P Y L}\right.$, per exposed $\mathrm{Ru}$ atom) is derived from the catalytic sequence proposed in PYL-B, Scheme 4, by considering the $b^{\text {th }}$ hydrogen addition event as the kinetically relevant step and applying pseudo steady-state assumptions on all reactive intermediates:

$r_{H Y D, P Y L}=\frac{k_{H Y D, P Y L}\left\{\left[R u^{4+}-\left(S H_{2}-P Y L\right)-S^{2-}\right]^{d}\left[R u^{4+}-(P Y L)-S^{2-}\right]^{1-d}\right\}_{b=1}\left[R u^{4+}{ }_{-}\left(H^{\delta-}\right)-S^{2-}-(H P Y L)\right]_{b=2}\left[R u^{4+}{ }_{-} S^{2-}-\left(H_{n-1} P Y L\right)\right]_{n=b, b \geq 3}}{\left[R u^{4+}-\left(H^{\delta-}\right)-S^{2-}-\left(H^{\delta+}\right)\right]}$

where $r_{H Y D, P Y L}\left(k_{H Y D, P Y L}\right)$ denotes the turnover rate (rate constant) for the kinetic relevant step, and [ $\left.T_{P Y L}\right]$ represents the total surface sites. Parameter $d$ equals 0 or 1 , depending on whether pyrrole adsorbs on site $\mathrm{i}$ [as $R u^{4+}-(P Y L)-S^{2-}, d=0$ ], or on site iii [as $\left.R u^{4+}-\left(S H_{2}-P Y L\right)-S^{2-}, d=1\right]$. Assuming that the coverages of the Ncontaining intermediates generated after the kinetic relevant step remain insignificant, the catalyst surfaces are covered with $R u^{4+}-S^{2-}, \quad R u^{4+}-\left(H^{\delta-}\right)-S^{2-}-\left(H^{\delta+}\right), \quad R u^{4+}-\left(S H_{2}\right)-S^{2-} \quad, \quad\left[R u^{4+}-(P Y L)-S^{2-}\right]_{b=1}^{1-d}$, 
$\left[R u^{4+}-\left(S H_{2}-P Y L\right)-S^{2-}\right]_{b=1}^{d},\left[R u^{4+}-\left(H^{\delta-}\right)-S^{2-}-(H P Y L)\right]$, and $\left[R u^{4+}-S^{2-}-\left(H_{n-1} P Y L\right)\right]_{n=b, b \geq 2}$. The total site balance is then expressed as:

$\left[T_{P Y L}\right]=\left[R u^{4+}-S^{2-}\right]+\left[R u^{4+}-\left(H^{\delta-}\right)-S^{2-}-\left(H^{\delta+}\right)\right]+\left[R u^{4+}-\left(S H_{2}\right)-S^{2-}\right]+\left[R u^{4+}-(P Y L)-S^{2-}\right]_{b=1}^{1-d}+$

$\left[R u^{4+}-\left(S H_{2}-P Y L\right)-S^{2-}\right]_{b=1}^{d}+\left[R u^{4+}-\left(H^{\delta-}\right)-S^{2-}-(H P Y L)\right]+\cdots+\left[R u^{4+}-S^{2-}-\left(H_{n-1} P Y L\right)\right]_{n=b, b \geq 2}$

The relative abundances of $R u^{4+}-(P Y L)-S^{2-}, R u^{4+}-\left(S H_{2}-P Y L\right)-S^{2-}$, and $R u^{4+}-S^{2-}-\left(H_{n-1} P Y L\right)$ are given as:

$$
\begin{aligned}
& {\left[R u^{4+}-(P Y L)-S^{2-}\right]=K_{P Y L, a d s, B}[P Y L]\left[R u^{4+}-S^{2-}\right]} \\
& {\left[R u^{4+}-\left(S H_{2}-P Y L\right)-S^{2-}\right]=K_{P Y L, a d s, A} K_{H_{2} S}\left[H_{2} S\right][P Y L]\left[R u^{4+}-S^{2-}\right]} \\
& {\left[R u^{4+}-\left(H^{\delta-}\right)-S^{2-}-(H P Y L)\right]=K_{H P Y L}\left(K_{P Y L, a d s, A}\right)^{d}\left(K_{P Y L, a d s, B}\right)^{1-d}[P Y L] K_{H_{2}}\left[H_{2}\right]\left[R u^{4+}-S^{2-}\right]} \\
& {\left[R u^{4+}-S^{2-}-\left(H_{n-1} P Y L\right)\right]} \\
& \quad=\left(K_{H_{n-1} P Y L} K_{H_{n-2} P Y L} \cdots K_{H P Y L}\right)_{n=b, b \geq 3}\left(K_{P Y L, a d s, A}\right)^{d}\left(K_{P Y L, a d s, B}\right)^{1-d}[P Y L]\left(K_{H_{2}}\left[H_{2}\right]\right)^{0.5 b-0.5}\left[R u^{4+}-S^{2-}\right]
\end{aligned}
$$

Substitution of Equations S11, S12, S20 and S26-S29 into Equation S25 gives:

$$
\begin{aligned}
& \frac{\left[T_{P Y L}\right]}{\left[R u^{4+}-S^{2-}\right]}=1+K_{H 2}\left[H_{2}\right]+K_{H 2 S}\left[H_{2} S\right]+\left\{K_{P Y L, a d s, B}[P Y L]\right\}_{b=1}^{1-d}+\left\{K_{P Y L, a d s, A} K_{H_{2} S}\left[H_{2} S\right][P Y L]\right\}_{b=1}^{d} \\
& +\left\{K_{H P Y L}\left(K_{P Y L, a d s, A}\right)^{d}\left(K_{P Y L, a d s, B}\right)^{1-d}[P Y L] K_{H_{2}}\left[H_{2}\right]\right\}_{b=2}+\cdots \\
& +\left\{\left(K_{H_{n-1} P Y L} K_{H_{n-2} P Y L} \cdots K_{H P Y L}\right)\left(K_{P Y L, a d s, A}\right)^{d}\left(K_{P Y L, a d s, B}\right)^{1-d}[P Y L]\left(K_{H_{2}}\left[H_{2}\right]\right)^{0.5 b-0.5}\right\}_{n=b, b \geq 3}
\end{aligned}
$$

$$
\text { If } \begin{aligned}
\varphi_{P Y L} & =\left\{K_{P Y L, a d s, B}[P Y L]\right\}_{b=1}^{1-d}+\left\{K_{P Y L, a d s, A} K_{H_{2} S}\left[H_{2} S\right][P Y L]\right\}_{b=1}^{d} \\
& +\left\{K_{H P Y L}\left(K_{P Y L, a d s, A}\right)^{d}\left(K_{P Y L, a d s, B}\right)^{1-d}[P Y L] K_{H_{2}}\left[H_{2}\right]\right\}_{b=2}+\cdots \\
& +\left\{\left(K_{H_{n-1} P Y L} K_{H_{n-2} P Y L} \cdots K_{H P Y L}\right)\left(K_{P Y L, a d s, A}\right)^{d}\left(K_{P Y L, a d s, B}\right)^{1-d}[P Y L]\left(K_{H_{2}}\left[H_{2}\right]\right)^{0.5 b-0.5}\right\}_{n=b, b \geq 3}
\end{aligned}
$$

Substituting Equations S11, S12, and S26-S31 into S24, the overall turnover rate of pyrrole is:

$r_{H Y D, P Y L}=\frac{k_{H Y D, P Y L}\left\{\left(K_{H_{n-1} P Y L} K_{H_{n-2} P Y L} \cdots K_{H P Y L}\right)_{n=b, b \geq 2}\left(K_{P Y L, a d s, A}\right)^{d}\left(K_{P Y L, a d s, B}\right)^{1-d}\right\}[P Y L]\left(K_{H_{2} S}\left[H_{2} S\right]\right)_{b=1}^{d}\left(K_{H_{2}}\left[H_{2}\right]\right)_{b \leq 2}^{b}\left(K_{H_{2}}\left[H_{2}\right]\right)_{b \geq 3}^{0.5 b+0.5}}{\left(\theta+\varphi_{P Y L}\right)^{2}}$

when $1 \leq b \leq 2$, Equation S32 becomes:

$r_{H Y D, P Y L}=\frac{\left.k_{H Y D, P Y L}\left\{\left(K_{H_{n-1} P Y L} K_{H_{n-2} P Y L} \cdots K_{H P Y L}\right)_{n=b, b \geq 2}\left(K_{P Y L, a d s, A}\right]\right)^{d}\left(K_{P Y L, a d s, B}\right)^{1-d}\right\}[P Y L]\left(K_{H_{2} S}\left[H_{2} S\right]\right)_{b=1}^{d}\left(K_{H_{2}}\left[H_{2}\right]\right)^{b}}{\left(\theta+\varphi_{P Y L}\right)^{2}}$

which is also Equation 18 of the main manuscript. 


\section{S4. Sensitivity Analyses on Kinetic Parameters.}

Figure S5 shows the sensitivity analyses performed on the parameters, including $K_{H 2 S}, K_{H P Y R, A} K_{P Y R, a d s} K_{H 2}$, $\left(K_{H P Y R, C}+1\right) K_{P Y R, a d s} K_{H 2 S} \quad, \quad k_{H Y D, P Y R, A} K_{H P Y R, A} K_{P Y R, a d s}\left(K_{H 2}\right)^{2} \quad, \quad k_{H Y D, P Y R, C} K_{H P Y R, C} K_{P Y R, a d s} K_{H 2 S} K_{H 2} \quad$ ， $k_{H Y D, P Y L} K_{P Y L, a d s, B} K_{H 2}$, and $K_{P Y L, a d s, B}$ obtained from non-linear regressions of the rate expressions for pyridine hydrogenation (Equation 17, Section 3.5) and pyrrole hydrogenation (Equation 18, Section 3.5), by introducing a change (e.g., $\pm 25 \%$ relative to the optimized value) to one of the parameters, and then calculating the resulting change in the sum of square of residues (relative to the optimized value) between the predicted and experimental values.
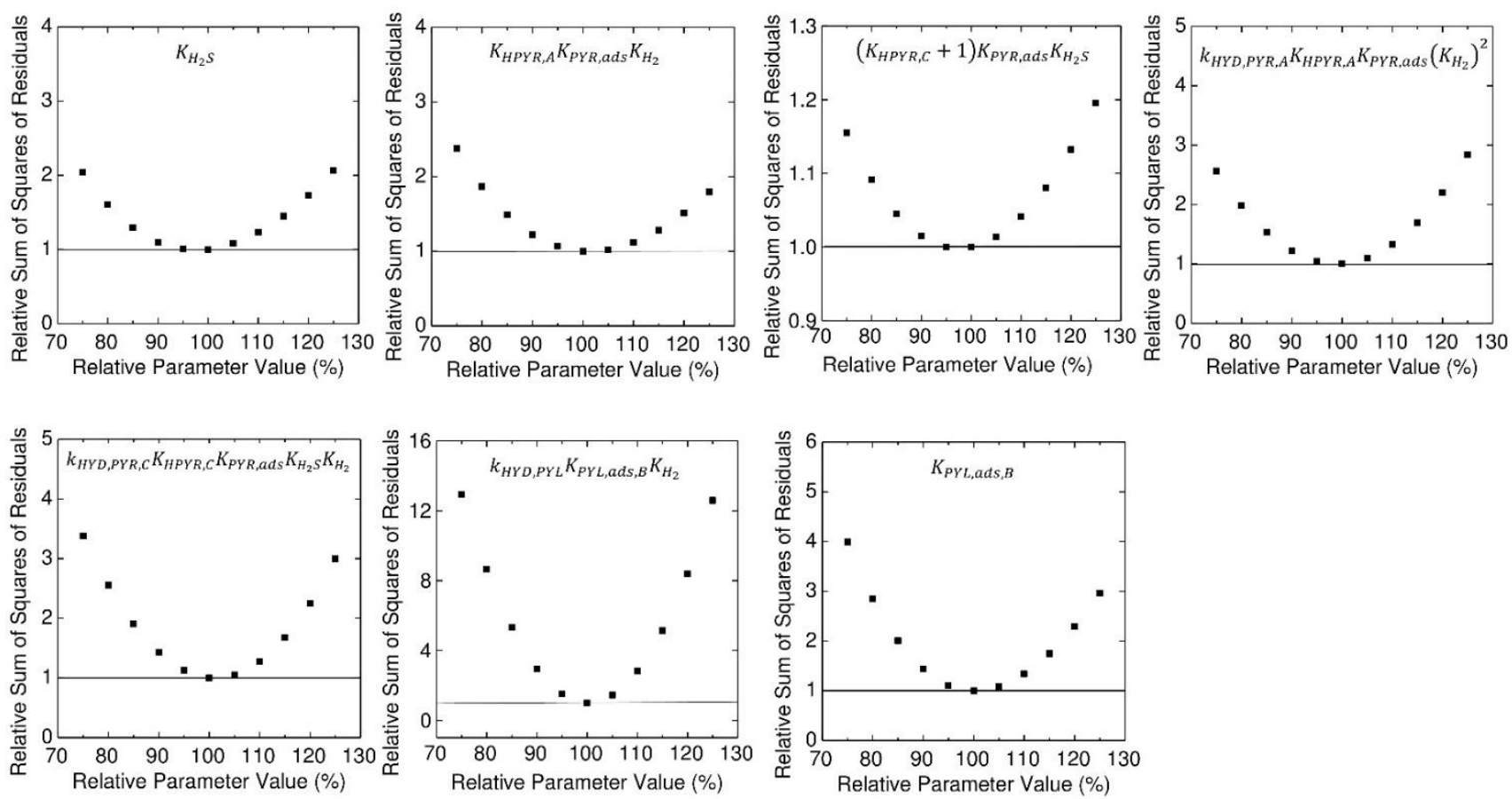

Figure S5. Sensitivity of sum of squares of residuals to variations in optimal parameters (Table 2) for $5.9 \mathrm{~nm} \mathrm{RuS}_{\mathrm{x}} / \mathrm{SiO}_{2}$ catalyst at $573 \mathrm{~K}$.

\section{S5. Surface Phase Diagrams Calculations.}

The relative stability of different $(\mathrm{RuS})_{\mathrm{x}} \mathrm{H}_{\mathrm{y}}$ surface termination models was determined through ab initio surface diagrams calculations. ${ }^{2}$ The Gibbs free energy of each surface slab $\left(\mathrm{G}_{\text {slab }}\right)$, including different surface terminations can be expressed as follows:

$G_{\text {slab }}=E_{\text {slab }}-N_{\mathrm{Ru}} \mu_{\mathrm{Ru}}-N_{S} \mu_{\mathrm{S}}-N_{\mathrm{H}} \mu_{\mathrm{H}}$ 
where $\mathrm{E}_{\text {slab }}$ is the total energy of the slab including a specific surface termination; $\mu_{\mathrm{Ru}}, \mu_{\mathrm{S}}$, and $\mu_{\mathrm{H}}$ are the ruthenium, sulfur, and hydrogen chemical potential, respectively; $\mathrm{N}_{\mathrm{Ru}}, \mathrm{N}_{\mathrm{S}}$, and $\mathrm{N}_{\mathrm{H}}$ are the number of ruthenium, sulfur, and hydrogen atoms included in the slab model.

Since the number of $\mathrm{Ru}$ atoms does not change in all the models considered, by choosing an appropriate reference state for $\mathrm{G}_{\text {slab }}$, it is possible to eliminate the dependence on $\mu_{\mathrm{Ru}}$. In this case we decided to use the surface model in which no sulfur vacancies and four surface hydrogen atoms are present $(\mathrm{S} 2+4 \mathrm{H})$, as the reference state. The new expression for $\mathrm{G}_{\text {slab }}$, considering the $\mathrm{S} 2+4 \mathrm{H}$ surface termination as the reference state would become:

$G_{\text {slab }}=\left(E_{\text {slab }}-\mathrm{E}_{\mathrm{S} 2+4 \mathrm{H}}\right)-\Delta \mathrm{N}_{\mathrm{S}} \mu_{\mathrm{S}}-\Delta \mathrm{N}_{\mathrm{H}} \mu_{\mathrm{H}}$

where $\mathrm{E}_{\mathrm{S} 2+4 \mathrm{H}}$ is the total energy of the $\mathrm{S} 2+4 \mathrm{H}$ surface model and $\Delta \mathrm{N}_{\mathrm{S}}$ and $\Delta \mathrm{N}_{\mathrm{H}}$ are the difference between the number of $\mathrm{S}$ and $\mathrm{H}$ atoms, respectively, present on a generic surface model and in the $\mathrm{S} 2+4 \mathrm{H}$ model. By expressing $\mu_{\mathrm{S}}$ and $\mu_{\mathrm{H}}$ in terms of $\mathrm{H}_{2} \mathrm{~S}$ and $\mathrm{H}_{2}$ chemical potentials ( $\mu_{\mathrm{H} 2 \mathrm{~S}}$ and $\mu_{\mathrm{H} 2}$, respectively), we obtain:

$G_{\text {slab }}=\left(E_{\text {slab }}-\mathrm{E}_{\mathrm{S} 2+4 \mathrm{H}}\right)-\Delta \mathrm{N}_{\mathrm{S}}\left(\mu_{\mathrm{H} 2 \mathrm{~S}}-\mu_{\mathrm{H} 2}\right)-\frac{1}{2} \Delta \mathrm{N}_{\mathrm{H}} \mu_{\mathrm{H} 2}$

with $\left(\mu_{\mathrm{H} 2 \mathrm{~S}}-\mu_{\mathrm{H} 2}\right)=\Delta \mathrm{E}_{\mathrm{el}}+\Delta \mathrm{E} \mathrm{ZPE}+\Delta \mathrm{H}(\mathrm{T})-\mathrm{T} \Delta \mathrm{S}(\mathrm{T})+\mathrm{RT} \ln \frac{P_{H 2 S}}{P_{H 2}}$, where $\Delta \mathrm{E}_{\mathrm{el}}$ stands for the difference between $\mathrm{H}_{2} \mathrm{~S}$ and $\mathrm{H}_{2}$ gas phase DFT total energies, $\Delta \mathrm{E}_{\mathrm{ZPE}}$ is the zero point vibrational energy term calculated through evaluation of the harmonic vibrational frequencies, $\Delta \mathrm{H}(\mathrm{T})$ and $\Delta \mathrm{S}(\mathrm{T})$ are the enthalpy and entropy differences between gas-phase $\mathrm{H}_{2} \mathrm{~S}$ and $\mathrm{H}_{2}$ calculated through Shomate equations following the procedure discussed in our previous studies, $;^{3-4} \mathrm{P}_{\mathrm{H} 2 \mathrm{~S}}$ and $\mathrm{P}_{\mathrm{H} 2}$ are the $\mathrm{H}_{2} \mathrm{~S}$ and $\mathrm{H}_{2}$ partial pressures, respectively. A similar expression can be derived for $\mu_{\mathrm{H} 2}$, in which the pressure dependence of $\mu_{\mathrm{H} 2}$ would be given by $\mathrm{P}_{\mathrm{H} 2}$ only.

\section{S6. Summary and Nomenclature of the Surface Models Employed and Complete Surface Phase Diagram.}

The top view of the unit cell of each model considered in this study is reported in the column named "Model". $\mathrm{Ru}, \mathrm{S}$, and $\mathrm{H}$ atoms are represented by green, yellow, and white spheres, respectively. Dashed grey lines delineate the unit cell lattice vectors. 


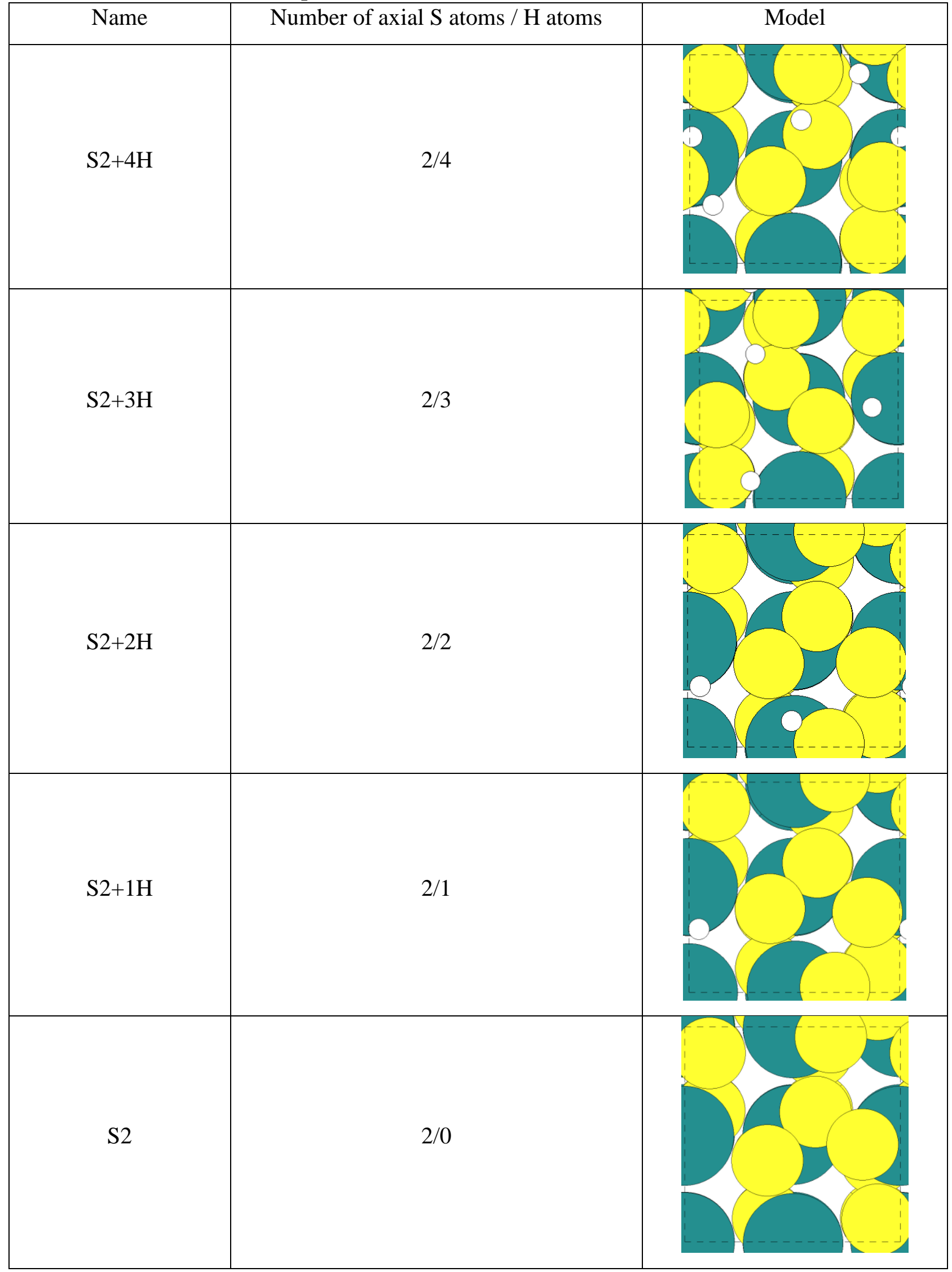


Table S4. Nomenclature and Top Views of S-Poor S1 Surface Models

\begin{tabular}{|c|c|c|}
\hline Name & Number of axial $\mathrm{S}$ atoms / $\mathrm{H}$ atoms & Model \\
\hline $\mathrm{S} 1+4 \mathrm{H}$ & $1 / 4$ & \\
\hline $\mathrm{S} 1+3 \mathrm{H}$ & $1 / 3$ & \\
\hline $\mathrm{S} 1+2 \mathrm{H}$ & $1 / 2$ & \\
\hline $\mathrm{S} 1+1 \mathrm{H}$ & $1 / 1$ & \\
\hline S1 & $1 / 0$ & \\
\hline
\end{tabular}


Table S5. Nomenclature and Top Views of S-Poor S0 Surface Models

\begin{tabular}{|c|c|c|}
\hline Name & Number of axial S atoms / $\mathrm{H}$ atoms \\
\hline $\mathrm{S} 0+2 \mathrm{H}$ & $0 / 2$ & \\
\hline $\mathrm{S} 0+\mathrm{H}$ & $0 / 1$ & \\
\hline
\end{tabular}

Table S6. Nomenclature and Top Views of S-Rich S4 Surface Models

\begin{tabular}{|c|c|c|}
\hline Name & Number of axial S atoms / $\mathrm{H}$ atoms \\
\hline $\mathrm{S} 4+2 \mathrm{H}$ & $4 / 2$ & Model \\
\hline
\end{tabular}




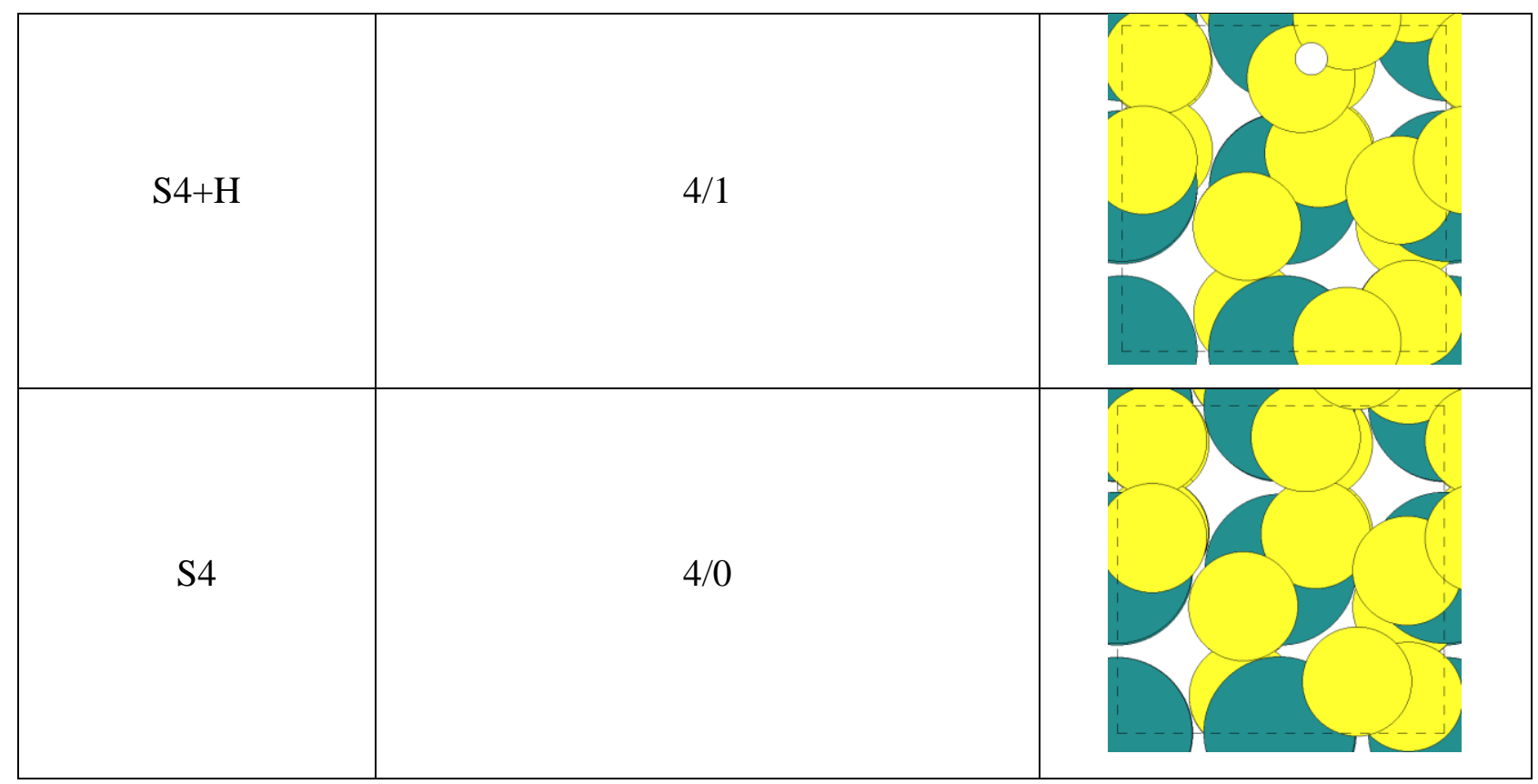

Table S7. Nomenclature and Top Views of S-Rich S3 Surface Models

\begin{tabular}{|c|c|c|}
\hline Name & Number of axial $\mathrm{S}$ atoms / $\mathrm{H}$ atoms & Model \\
\hline $\mathrm{S} 3+2 \mathrm{H}$ & $3 / 2$ & \\
\hline $\mathrm{S} 3+\mathrm{H}$ & $3 / 1$ & \\
\hline S3 & $3 / 0$ & \\
\hline
\end{tabular}


The complete surface phase diagram including all the investigated surface models, presenting different terminations, is reported in Figure S6.

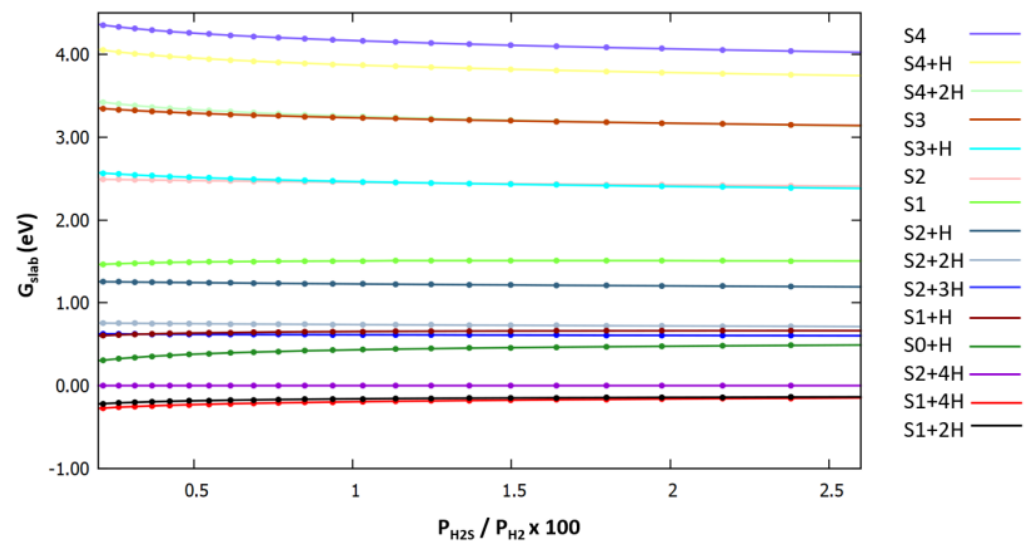

Figure S6. Calculated variation of Gibbs free energy $\left(\mathrm{G}_{\mathrm{slab}}\right)$ of the least stable models of hydrogenated RuS 2 (100) surface with respect the $\mathrm{P}_{\mathrm{H} 2 \mathrm{~S}} / \mathrm{P}_{\mathrm{H} 2}$ ratio. The interval spanned in the $\mathrm{P}_{\mathrm{H} 2 \mathrm{~S}} / \mathrm{P}_{\mathrm{H} 2}$ ratio is consistent with the operating conditions for pyridine and pyrrole hydrogenation. The stable $\mathrm{S} 1+4 \mathrm{H}, \mathrm{S} 2+2 \mathrm{H}$, and reference $\mathrm{S} 2+4 \mathrm{H}$ surface models are reported for the sake of comparison.

\section{S7. Adsorption Configuration of Pyrrole on the S1+2H Surface Model.}

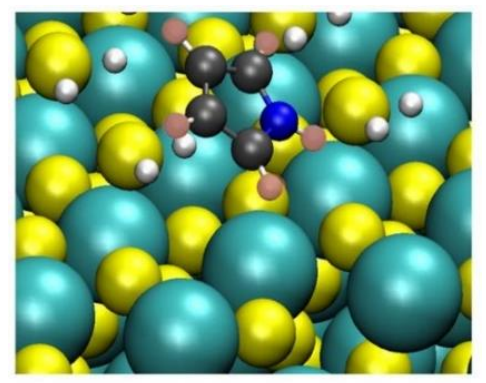

PYL B*

$E_{\text {ads }}=-0.64 \mathrm{eV}$

$\mathrm{NH} \cdots \mathrm{S}^{2-}=2.52 \AA$

Figure S7. Optimized structures, adsorption energy and relevant bond lengths of pyrrole (PYL B*), on the S1+2H surface model. Ru, S, N, C, and $\mathrm{H}$ atoms are represented by cyan, yellow, blue, grey, and white spheres, respectively. Additionally, the pink hydrogen atoms belong to the aromatic ring, while white ones to the surface.

\section{S8. Surface Deprotonation Energy (SDE) Definition.}

The acidity of each surface termination was evaluated through the use of SDE. This can be defined as the energy required to complete the following process: 
$(R u S)_{x} H_{y}+P Y R(P Y L)_{g a s} \rightarrow(R u S)_{x} H_{(y-1)}+e^{-}{ }_{\text {surf }}+P Y R H^{+}\left(P Y L H^{+}\right)_{g a s}$

where $(R u S)_{x} H_{y}$ is a generic surface slab model with $y \mathrm{H}$ atoms coordinated to $\mathrm{Ru}$ or $\mathrm{S} ; P Y R(P Y L)_{\text {gas }}$ is pyridine (pyrrole) in gas phase. $(R u S)_{x} H_{(y-1)}$ is the same surface slab previously defined, in its deprotonated form; $e^{-}$surf is the electron formed during $(R u S)_{x} H_{y}$ deprotonation process, while $P Y R H^{+}\left(P Y L H^{+}\right)_{g a s}$ is the gas phase energy of pyridine (pyrrole) in its protonated form. In other words, SDE is the energy required (or gained) for deprotonating one of the surface terminations and protonating pyridine or pyrrole in the gas-phase. Thus, SDE, differently from binding energies, would vary depending on the chemical nature of protonated molecule and that of the surface termination considered.

To estimate SDE, the following thermodynamic cycle was used:

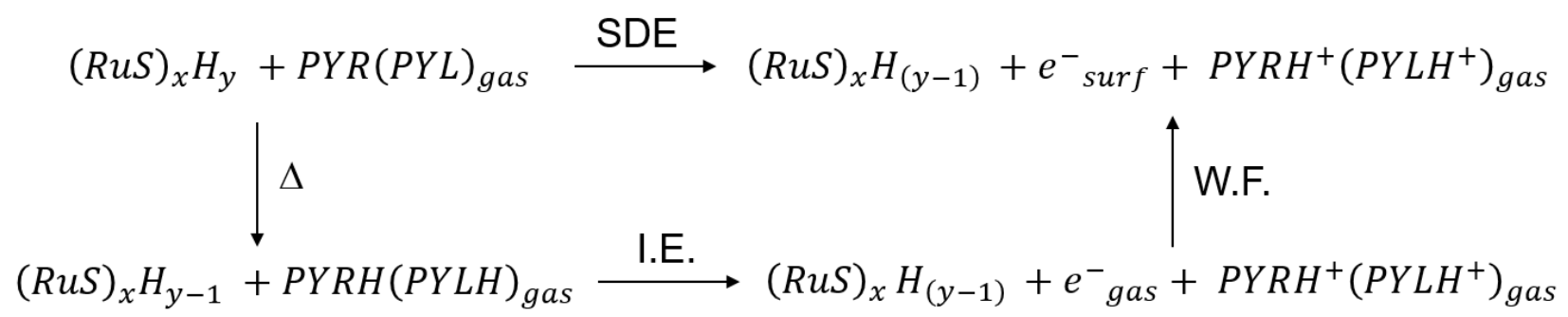

where $\Delta=\left(\mathrm{E}_{(\mathrm{RuS}) \times \mathrm{Hy}-1}-\mathrm{E}_{(\mathrm{RuS}) \times \mathrm{xH}}\right)+\left(\mathrm{E}_{\text {PYRH(PYLH)gas }}-\mathrm{E}_{\text {PYRH(PYLH)gas }}\right)$. All the reported quantities are DFT energies associated to the system defined in the respective energy term subscript; I.E. is the ionization energy of PYRH or PYLH in the gas phase, calculated at the same level of theory described in the Computational Methods section of the main text. To identify the most stable protonated form of PYR and PYL, the energy of all the possible isomers of PYRH and PYLH was calculated in the gas phase. The results showed that pyridine-1-ium and 2-H-pyrrole are the most stable isomers of PYRH and PYL, respectively. W.F. is the work function of each surface model considered in the evaluation of SDE. Work function were estimated through the calculation of plane averaged electrostatic potential of each slab adapting the methodology previously proposed by Fall et al. ${ }^{5}$ For W.F. calculations, the inclusion of at least $35 \AA$ of vacuum and slab dipole corrections was ensured. Charged unit cells, sufficiently large ( $51 \AA \times 50 \AA \times 49 \AA$ ) to ensure the convergence of the energy with respect to the unit cell size, and a neutralizing background charge were used. With these settings, the ionization energies (I.E.) of PYR and PYL are 8.88 and $7.79 \mathrm{eV}$, in reasonable agreement with those determined from experiments of 9.26 (PYR) and $8.21 \mathrm{eV}$ (PYL), respectively. ${ }^{5}$ Moreover, the calculated difference in I.E. between PYR and PYL ( $\triangle$ I.E. $=1.09$ $\mathrm{eV})$ is in close agreement with the experimental value $(\Delta \mathrm{I}$.E.exp $=1.05 \mathrm{eV})$. Thus, our computational parameters should be appropriate for capturing differences in SDEs arising from the I.E. contribution. 


\section{S9. Equivalence of Hydrogens in the $\mathrm{Ru}^{4+}-\mathrm{SH}_{2}$ Surface Chemical Moiety.}

As demonstrated in the main text by means of surface phase diagram calculations, the $\mathrm{S} 1+4 \mathrm{H}$ surface model, showing $\mathrm{Ru}^{4+}-\mathrm{H}^{\delta-}, \mathrm{S}^{2}-\mathrm{H}^{\delta+}$, and $\mathrm{Ru}^{4+}-\mathrm{SH}_{2}$ surface moieties is the most stable under our experimental reaction conditions. Each of the hydrogens is characterized by a different Bader charge, which reflects their chemical properties. According to Bader charge analysis, the two hydrogen atoms appearing in $\mathrm{Ru}^{4+}-\mathrm{SH}_{2}$ have a slightly different Bader charge of $+0.07\left|\mathrm{e}^{-}\right|$and $+0.15\left|\mathrm{e}^{-}\right|$(Scheme 1). However, this difference can be attributed to the local chemical environment in which each hydrogen experiences in its immediate surroundings, a consequence of the static nature of DFT calculations employed here. To corroborate our assumption on the chemical equivalence of these two hydrogen atoms we calculated the activation energy barrier associated to the scrambling between these two hydrogens, labeled $\mathrm{H}^{\mathrm{I}}$ and $\mathrm{H}^{\mathrm{II}}$ in Figure $\mathrm{S} 8$. This process is equivalent to rotating the $-\mathrm{SH}_{2}$ chemical group along the $\mathrm{Ru}-\mathrm{S}$ bond, which would bring $\mathrm{H}^{\mathrm{I}}$ in the position of $\mathrm{H}^{\mathrm{II}}$ and vice versa, as shown in Figure S8. The activation energy barrier for the rotation of $-\mathrm{SH}_{2}$ along the $\mathrm{Ru}-\mathrm{S}$ axis was calculated using the Nudged Elastic Band (NEB) method, ${ }^{6}$ by scanning the rotation angle $\theta$ showed in Figure S8. An activation energy barrier of $0.09 \mathrm{eV}$ was calculated, demonstrating the easy rotation of the $-\mathrm{SH}_{2}$ chemical moiety, and the associated scrambling of the two hydrogens. Therefore, the properties of $\mathrm{H}^{\mathrm{I}}$ and $\mathrm{H}^{\mathrm{II}}$ would be averaged at the reaction conditions, and their chemical identity would be indistinguishable.
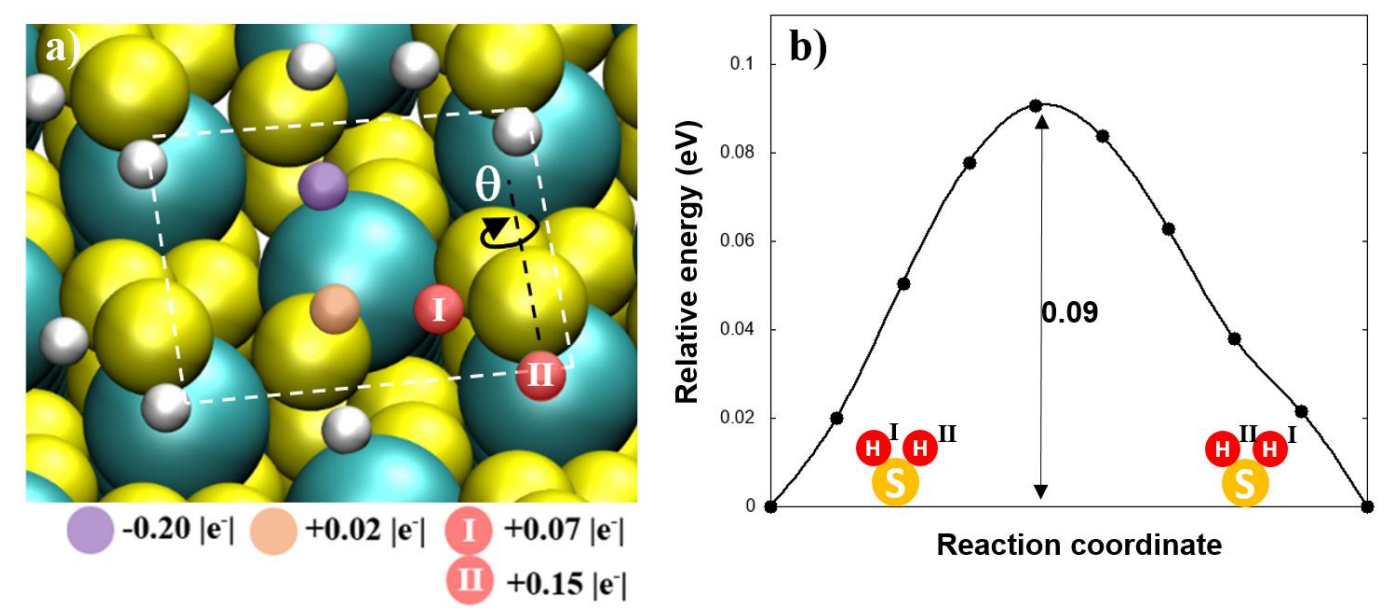

Figure S8. Chemical equivalence of hydrogens in $\mathrm{Ru}^{4+}-\mathrm{SH}_{2}$. (a) Optimized geometry of the $\mathrm{S} 1+4 \mathrm{H}$ surface model (tilted view); $\mathrm{Ru}, \mathrm{S}$, and $\mathrm{H}$ atoms are represented by cyan, yellow, and white spheres, respectively. Red, brown, and purple shaded hydrogen atoms belong to $-\mathrm{SH}_{2},-\mathrm{SH}$, and $\mathrm{Ru}-\mathrm{H}$ surface terminations, respectively. Bader charge of each surface hydrogen is reported using the same color-code. Dashed white lines delineate the unit cell lattice vectors. The scrambling between $\mathrm{H}^{\mathrm{I}}$ and $\mathrm{H}^{\mathrm{II}}$ can be obtained by rotating the $-\mathrm{SH}_{2}$ chemical moiety along the $\mathrm{Ru}-\mathrm{S}$ axis (dashed black line) by varying the $\theta$ angle . (b) Activation energy barrier associated to the $-\mathrm{SH}_{2}$ rotation along the $\mathrm{Ru}-\mathrm{S}$ bond axis. In the initial and final state, $\mathrm{H}^{\mathrm{I}}$ and $\mathrm{H}^{\mathrm{II}}$ switch their position, as shown in the cartoon in the inset. 
S10. Summary of Differential Hydrogen Binding Energies $(\Delta B E)$ of the $S 1+4 H$ Surface Model.

Table S8. Calculated Differential Hydrogen Binding Energies $(\Delta B E)$ for Each Hydrogen Site on the S1+4H Surface Model

\begin{tabular}{|c|c|c|c|}
\hline \multirow{2}{*}{ Number of surface $H$} & \multicolumn{3}{|c|}{$\Delta B E(e V)$ hydrogen sites $^{a}$} \\
\cline { 2 - 4 } & site ii + & site ii- & site iii \\
\hline $\mathbf{4}$ & $\mathbf{2 . 5 6}$ & 2.94 & 2.95 \\
\hline $\mathbf{3}$ & - & $\mathbf{1 . 9 4}$ & 2.93 \\
\hline $\mathbf{2}$ & - & - & $\mathbf{3 . 0 4}$ \\
\hline
\end{tabular}

${ }^{a}$ Starting with the completely hydrogenated surface $(4 \mathrm{H})$, the $\Delta \mathrm{BE}$ has been calculated for each hydrogen chemical moiety. The one with the lowest $\triangle \mathrm{BE}$ (in bold), the weakest bound, is removed and $\triangle \mathrm{BE}$ is recalculated on the remaining hydrogen atoms to assess the order in which they are deprotonated. The weakest bound at each level of surface hydrogenation are reported and commented in Scheme 1c of the main text.

\section{References}

(1) Van Hardeveld, R.; Hartog, F., The Statistics of Surface Atoms and Surface Sites on Metal Crystals. Surf. Sci. 1969, 15, 189-230.

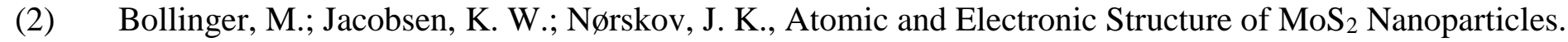
Phys. Rev. B 2003, 67, 85410-85426.

(3) Gokhale, A. A.; Kandoi, S.; Greeley, J. P.; Mavrikakis, M.; Dumesic, J. A., Molecular-Level Descriptions of Surface Chemistry in Kinetic Models Using Density Functional Theory. Chem. Eng. Sci. 2004, 59, 4679-4691.

(4) Grabow, L.; Mavrikakis, M., Mechanism of Methanol Synthesis on $\mathrm{Cu}$ through $\mathrm{CO}_{2}$ and $\mathrm{CO}$ Hydrogenation. ACS Catal. 2011, 1, 365-384.

(5) Fall, C.; Binggeli, N.; Baldereschi, A., Deriving Accurate Work Functions from Thin-Slab Calculations. J. Phys.: Condens. Matter 1999, 11, 2689-2696.

(6) Henkelman, G.; Uberuaga, B. P.; Jónsson, H., A Climbing Image Nudged Elastic Band Method for Finding Saddle Points and Minimum Energy Paths. J. Chem. Phys. 2000, 113, 9901-9904. 